\title{
Customer Focused Advertising Through Server Based Broadcast
}

Blake E. DeBray, University of Central Missouri, USA

Mustafa Kamal, University of Central Missouri, USA

\begin{abstract}
Advertising has become a common part of our culture and is an area that is undergoing continuous changes. The same can be said for the means of distributing advertisements. Television and radio are no longer the only means of media distribution. New technologies have allowed consumers to view their favorite programs (and the advertisements that come with them) from a wide variety of devices and methods. Content can be viewed from home, out in public and on the road in ways that were previously unimaginable. For several decades, the most common way to reach viewers was through the television. Although this method of distribution will not change any time soon, other new ways to reach audiences have sprung from modern technologies. What does this mean for the businesses who have ruled the advertising industry for all of these years?
\end{abstract}

\section{CHANGES IN ADVERTISING}

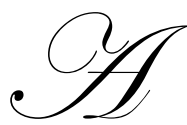

dvertising and ad-related businesses have grown drastically over the years; however, there have recently been technological and economic forces with large scale impacts on the industry. The economic downturn has resulted in reduced spending for many businesses, including advertisers. For example, the automotive industry, a major contributor to advertisement revenue, drastically reduced spending due to the economic downturn. The total amount of spending for the automotive industry dropped $13.5 \%$ alone in 2007 [1]. As a result, businesses such as television stations felt the impact in sales and had to reconsider their own cost structure. Another change is the result of the consumer behavior. People tend to spend less time in front of the television and more in front of computers and mobile electronic devices. Advertisers are becoming more concerned with viewers pre-recording programs and skipping commercials. Advertisers are worried that their ability to reach audiences as in the past is shrinking with traditional distributors such as broadcast TV. With cable, broadcast, satellite, and other digital means, audience fragmentation has grown radically. Such concerns have resulted in advertisers and content distributors to look for new ways to increase revenue while becoming more efficient.

These changes become apparent when looking at ad spending trends. A report provided by In-Stat states, "The key trend for all US advertising is the move away from print and into electronic forms. Within the electronic forms, the trend is to move away from the traditional mass marked delivery services and move toward more personalized and relevant delivery services". [2]

In another recent 2009 market study produced by a leading market research company Research and Markets [3], reports that Nationwide Broadcast Networks and Subscription TV Networks will continue to be the best delivery vehicles for mass market TV advertising. However, advertising that targets identifiable ethnic, cultural, language or special-interest audiences will see above-average growth rates through 2012. Geographic advertising is expected to grow to about 30\% of US TV ad spending. -Hardware-Based Addressable Advertising solutions already exist on the Internet, and its' impact will be growing on a market-by-market basis. audiences.

These trends indicate that advertisers are becoming more focused on accurately targeting specific 


\section{Addressable Advertising}

Newer technologies not only allow for new ways to reach audiences, it also allows for specific targeting of viewers. The concept of addressable advertising is the ability for advertisers to "create a profile of the type of person they would like to have view their ad, and then a digital delivery system delivers the ad each time an opportunity arises to reach people who match the profile" [2]. Traditionally, TV advertising was directed to a mass market with multiple advertising messages to a wide variety of viewers. Until recently, the ability to target a very narrow and specific group of viewers was very difficult, if not impossible. The most common form of addressable advertising has been at the geographical level. Local stations would sell airtime to businesses in the area and support local promotional programming that would reach audiences within the same physical location. Subscription TV networks are able to take addressable advertising in a different direction, by providing specialty channels such as Sifi and the History Channel in order to target a specific niche. Other channels target specific ethnic and language groups. These specialty stations allow advertisers to reach a specific viewer profile that is more likely to watch specialty channels than other viewers.

Internet and Video on Demand is capable of taking addressable advertising to an entire new level due to the ability of two-way communication of viewer behavior. Based on the amount of information provided of the viewer, advertisements in these mediums could be displayed to a user based on their geographic location, demographic category (male, female, age, etc), and possibly more personal information (likes and dislikes). Internet tracking methods could also determine which advertisements to show based on recently visited web sites and any common behavior of the user.

Addressable advertising has always been a topic of high interest for advertisers, but it has become a bigger issue due to the recent economic difficulties. With less spending available, advertisers are expecting more bang for their buck. These concerns are directly related to the distribution methods that are used to reach viewers. The ability to target a specific viewer profile varies from one medium to another.

\section{Advertising Distribution Methods}

As mentioned before, technology has brought newer content distribution methods and new ways to advertise. Viewers are now capable of accessing content from their computers, cell phones and other devices. Technology has also helped improve existing models, such as broadcast TV and cable. TV stations and cable companies were previously limited to the television, but have recently ventured into other distribution means. The common theme for all content distribution methods is reaching targeted audiences in new ways. How this is done varies from method to method.

\section{ADVERTISING ON THE INTERNET}

The internet is one such method that has made an impressionable impact on the advertising industry. Not only does the internet provide advertisers with a new outlet to reach audiences, it also allows content providers to distribute programming and gain additional ad revenue as well. Essentially, all distribution forms utilize the internet in one way or another. Radio and Television stations build websites that allow users to access programming and locally targeted content. This also allows stations to introduce an additional outlet for advertising. Video on Demand can be distributed on the internet, allowing any consumer with a computer to access content and the advertisements included therein.

Internet advertising has resulted in many changes and noticeable differences when compared to the more traditional means of content and advertiser distribution. One major difference between the internet and broadcast model is the elimination of time boundaries. A television station is limited in the amount of advertisements it can sell in a twenty-four hour window, whereas the internet is not so linear. Internet advertising is based on the amount of users that access content instead of the time in which the content runs. Also, unlike television, web pages can display multiple advertisements and information at any given time. These changes allow website owners to sell more advertising spots than on television. Internet advertising also has the ability to collect accurate audience metrics via two-way tracking through cookies and other methods [see fig-1]. Tools such as DoubleClick keep track 
of the number of times an advertisement is viewed or clicked on. This allow advertisers to get realistic and accurate data on the audience reach and effectiveness of the advertisements. It also better supports user-specific addressable advertising. Eventually, this could provide long-term tracking of user behavior in order to determine which ads the viewer is most likely to respond to.
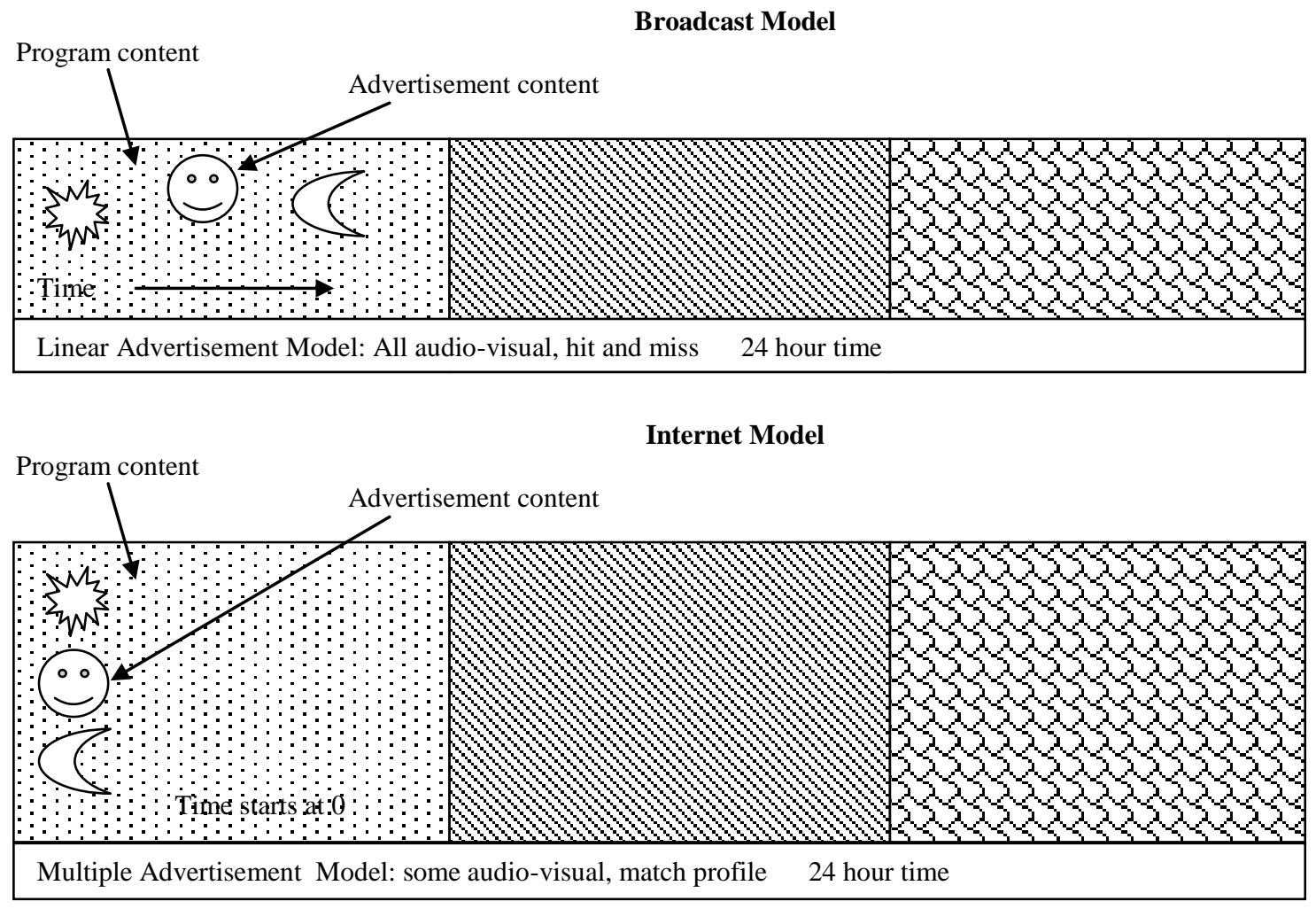

Figure 1

Internet advertising has been around for some time now, but it initially grew slowly. The technology to provide the same content over the internet that is available on TV did not exist until recently, which resulted in slower advertisement sales than in television. Internet advertising needs to continue to develop in order for it to be able to become a strong, independent competitor to other models.

\section{Video On Demand}

Video on Demand (V.O.D.) is a fairly new medium, but is quickly growing. This new form of distribution allows users to search for the specific programming that they are interested in and watch it at their own leisure. The concept of V.O.D. is actually presented in several models. Cable and satellite companies provide subscribers video on demand content with a "pay as you watch" service, while websites like Hulu provide content for free with ad insertions.

Sites that provide V.O.D. content, particularly Hulu, definitely show promise. In less than a year, Nielsen indicated that "Hulu became the Internet's second-most-popular video site, trailing only YouTube" [4]. The content selection on Hulu is broad as well. With supporters like NBC Universal, Fox, Disney and the use of over 150 content providers, viewers are likely to find their favorite shows like "Lost" and watch them at their choosing. Since viewers go to Hulu looking for a specific program, it allows them to charge advertisers a premium on spots. For example, it costs an advertiser sixty dollars per thousand viewers on Hulu or TV.com for spots within an episode of 
The Simpsons. The same advertisement on TV would cost around twenty to forty dollars per thousand viewers [5]. The higher cost is worth it for most advertisers, as they typically end up reaching the audience number that they were guaranteed. Another interesting finding is that since there are less advertisements in a program on Hulu, "viewers are twice as likely to remember a commercial they've seen on Hulu than on television".

Unlike TV, the video on demand model has not truly been finalized. In fact, cable providers have recently discussed providing web-based content as part of their subscription fees instead of charging per viewing. Services such as TV Everywhere could allow subscribers to view content online at no additional cost. The idea is unique since it allows you to "pay once and you can access the complete range of content-from HBO to TNT to Sci-Fi Channel—on any device, through any delivery mechanism" [6].

A major downside to sites such as Hulu is that internet V.O.D. is commonly viewed on the computer, whereas "TV is more of a sit-back medium" [7]. Another issue is the inability to promise advertisers a slot within a specific program. Hulu has to offer a slightly lower rate due to this limitation. Hulu also has issues with unsold inventory. "Because Hulu is growing fast, it's intrinsically not sold out, and agencies feel little pressure to lock in that inventory beforehand" . Even with the limitations, the capabilities of stand-alone V.O.D. services such as Hulu is promising.

\section{Subscription TV}

Cable and Satellite companies have seen strong growth in recent years and, until recently, has been the sole competitors in video distribution. Subscription TV networks is one of the few mediums showing growth in ad revenue [2]. Cable and Satellite networks have not been affected as much by the cut in advertising revenue due to the viewer subscription fees.

The Cable TV industry has promised to simplify addressable advertising at the geographic level by introducing Project Canoe. The concept would allow advertisers to purchase air time within a specific program at a specific time of day, all while indicating the desired number of viewers to reach in a given geographic location, regardless of what cable provider service is available in the area.

\section{Advertising for Broadcasters}

Newer technologies have benefited broadcasters as well. The introduction of digital broadcasting not only allows stations to supply high-definition programming on their main feed, but it has also "allowed stations to supply multiple programming feeds on their bandwidth" [2008]. This provides stations with the flexibility to supply additional content to gain more audiences. Additional channels can also allow broadcast groups to target specific audiences. For example, many stations are supplying 24-7 weather, multi-lingual, and location-specific channels. The extra bandwidth could supply broadcasters with more revenue due to the added airtime from the new feeds. Creating a channel that is targeted to a specific audience can attract new advertisers who wish to reach specific groups. It could also allow stations to better guarantee reaching a targeted demographic, resulting in better rates.

The major difference between broadcast TV and other mediums is the business model. For television, the model is supported by presenting content at a set timeframe and for a specific device: the television. With the introduction of computers, multimedia devices and the latest cell phones, the device limitation is slowly becoming more apparent. As mentioned earlier, changes in audience behavior will most likely increase the value of such devices for receiving video content. Another difficulty with the broadcast model is the changes with content providers. Websites like Hulu allow networks to distribute content directly, instead of supplying it through affiliates. Some networks, like ABC, host websites of their own; this also allows them to distribute content directly, as well as generate advertising revenue.

In addition to other mediums, newer technologies within the broadcast industry itself have caused some issues with advertisers. The introduction to digital video recorders (DVR) such as TiVo as well as cable provided DVRs allow viewers to record their shows and skip advertisements that were otherwise unavoidable. DVRs make it difficult for audience tracking, which results in less accurate results. The combination of DVRs and the lack of 
accurate viewer metrics have resulted in a loss in faith from advertisers.

The transition in technology and introduction in new models have caused trouble for the broadcast model; however, there is one thing for sure that has always greatly benefited broadcasters: the broadcast model is proven. Unlike the newer mediums, the broadcast model has been in use for a long time and is something that content providers and advertisers easily understand. Such an advantage can give the broadcasters the time that they need to adjust, and quite possibly allow them to develop the next model for all media distributors.

\section{Personalized TV}

Another new concept being introduced by Sezmi promises "personalized TV" that provides the benefit of subscription television that is found in cable and satellite and is presented in a viewer-selected format that is found in video on demand and the internet. Sezmi promises to provide viewers with a wide variety of content through both broadband and over-the-air means. The media that viewers will have access to could include "live TV, prerecorded content on the DVR, 'browse and request' archived video, pay-per-view, and Web content". The idea of personalized TV not only gives the viewer the content they wish to see, but also provides a more interactive experience in both programming and advertisements.

Personalized TV is too new to be sure if it will succeed, but the model could be the best of all worlds. The incorporation of interactive content and two-way metric tracking appears to be the model that all distribution mediums are trying to reach. If products such as Sezmi can succeed, then there is a good chance that Personalized $\mathrm{TV}$ can become a very effective content and advertising distribution method in the future.

\section{MODEL FOR TARGETED ADDRESSABLE ADVERTISING}

All of the previously mentioned models are striving for similar goals, such as improved audience reach and targeting, and improved efficiency. Unfortunately, each model proposes this solution in a different way. Suppose there was a platform in place that could distribute content from a variety of providers, target advertisements for specific groups or viewers, and support multiple viewing devices. Such a model could reduce cost for content providers, distributors and advertisers, all while giving audiences a wider variety of content and viewing flexibility. Figure 2 indicates how such a platform could accomplish these goals.

The distribution platform would contain a network of distribution centers, which store and transmit the media. A Centralized Distribution Center would exist in order to allow content providers and advertisers to submit media to a single location. The Centralized Distribution Center would also provide viewing metrics to advertisers, content providers, and ratings systems such as Neilson.

Local Distribution Centers would exist within each Designated Market Area (DMA). These centers would store content requested from the Centralized Distribution Center, as well as any locally generated media. Each Local Distribution Center would contain advertisements obtained from the Centralized Distribution Center (national advertisements) and from local advertisers. The Local Distribution Center would also contain an advertisement placement server, which could dynamically place. Advertisements in real-time based on the current viewer and the advertisement library.

The Local Distribution Centers are also responsible for sending the content and advertisements to the viewers. The content could be distributed over-the-air via transmitters or digitally by broadband and cable. A wide variety of viewing devices could then receive the content instead of being limited to the television or computer. Televisions that utilize a set-top box and computers could store programming for later use. Personal devices, such as cell phones, could pick up content on the fly for immediate viewing. In both cases, there would be constant communication between the device and the Local Distribution Center in order to record viewing data and place usertargeted advertisements in real-time. Viewers will also benefit by being able to access a wide variety of content at any time, from any device. 


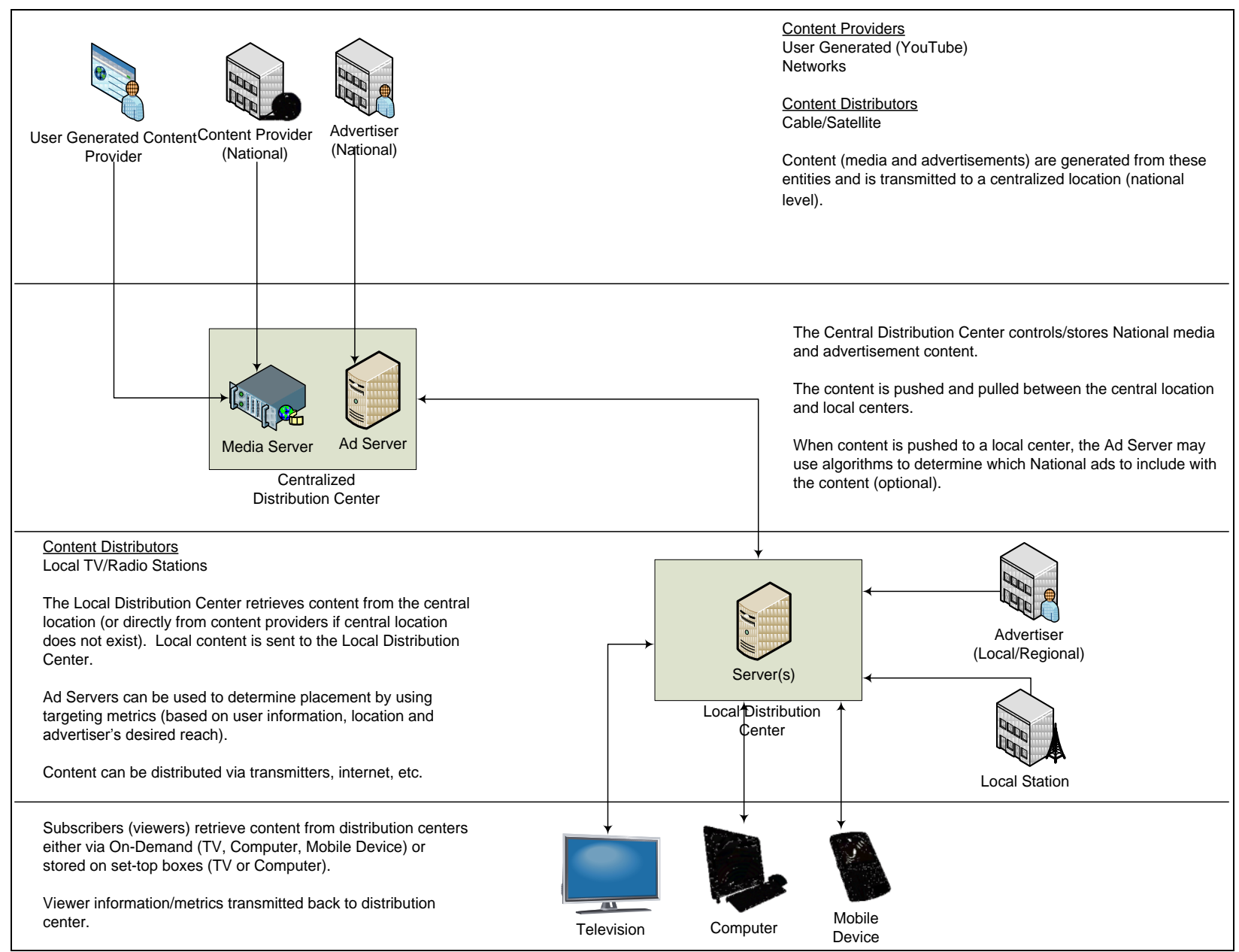

Figure 2 - Content Distribution Method

\section{Content Providers}

The proposed model would support a wide variety of content providers. Networks such as NBC and Fox could distribute their content nationally by sending media to a centralized distribution center. The model would potentially be capable of distributing user-generated content from providers such as YouTube. The media would flow to a Centralized Distribution Center, where it would remain on a set of media servers for immediate or future use. Local Distribution Centers would request the media as needed, or the content can be "broadcasted" live. Viewing metrics for the individual pieces of content are returned to the Centralized Distribution Center, which can then be sent to each content provider. The ending result would allow accurate viewer tracking, allowing content providers to better determine the popularity of each program.

\section{Content Distributors}

The proposed platform would not replace content distributors, but allow a common workflow of content and advertising distribution. The idea is to ensure that the distribution platform is flexible enough to support multiple scenarios and be adaptive to changing market trends and technologies. 


\section{CONCLUSION}

The future does not necessarily look grim for any distribution method, as long as they learn to adapt to the changing market conditions, expectations of advertisers and the behavior of audiences. Broadcast TV needs to focus on improving the capability to target specific audiences past the geographic level as well as incorporate the benefits of the other models. Cable also needs to improve addressable advertising and come to a common solution on a V.O.D. model in order to compete with Hulu and TV.com. Hulu continues to grow and gain supporters. If they can improve ad placement within specific programs and viewer targeting, as well as close the gap between internet V.O.D. and the television, then Hulu should continue to prosper. Personalized TV is a brand new concept. Sezmi is currently being tested in limited markets. It will take some time for this new, unique model to establish itself; however, it could have great potential. The proposed distribution platform in this paper could benefit content distributors, content providers, advertisers and viewers by providing a common distribution method that is flexible and accurate. It attempts to close the gaps in all existing models, such as limited addressable advertising capabilities, advanced advertisement tracking and viewer metrics and support for a wide variety of viewer devices. It also adds the benefit of non-linear based advertising, as any number of viewers watching the same program would most likely view a different set of advertisements. In essence, the television model and internet model could merge, providing the benefits of both to all participants in the model.

Economic, viewer and technology changes have drastically impacted advertisers and content distributors. Advertisers are attempting to be more cost effective by spending less and expecting a more targeted audience reach with what they do spend. Distributors are trying to adjust their models to have a larger audience reach, as well as better support addressable advertising. Everyone knows what is needed to succeed in the changing market; however, they have not yet decided on the best solution. It is quite possible that there will not be a single answer, and all distribution methods are here to stay for now. No matter the challenges, one thing is for certain. These changes have shown an adaptable industry, one which will remain a part of our society in the foreseeable future.

\section{REFERENCES}

1. Semida, Katerina. (n.d.). Decline in Television Advertising. Retrieved June 24, 2009, from wikinvest: http://www.wikinvest.com/concept/Decline_in_Television_Advertising.

2. In-Stat. (2008). Addressable Advertising Slowly Arrives. Scottsdale, AZ: Kaufhold, Gerry.

3. Research and Markets, Guinness Center, Dublin, Ireland http://www.researchandmarkets.com/reports/616008

4. O'Leary, Noreen. (2009). Searching for Life on Hulu, [Abstract], MediaWeek, v.19 (21), p5-7. Retrieved from EBSCO Host database.

5. Paul, Ian. (2009). The Simpsons: Worth More on Hulu than Fox. PCWorld. Retrieved 06/30/2009. From http://www.pcworld.com/article/167344/the_simpsons_worth_more_on_hulu_than_fox.html.

6. $\quad$ Anderson, Nate. (2009). "TV Everywhere": pay your cable bill, watch Entourage online. Retrieved 07/01/2009 from http://arstechnica.com/media/news/2009/03/tv-everywhere-pay-your-cable-bill-watchentourage-online.ars.

7. Hampp, Andrew \& Learmonth, Michael. (2009). What happened to all that talk about online? Nets rally around TV [Abstract]. Advertising Age, 80 (19), p3-21. Retrieved from EBSCO Host database.

8. $\quad$ Romano, Allison. (2008). Local Stations Multiply. Broadcasting \& Cable, p16-18. 


\section{NOTES}

